\title{
Myocardial fibrosis in desmin-related hypertrophic cardiomyopathy
}

\author{
Yi He${ }^{1}$, Zhaoqi Zhang ${ }^{1 *}$, Daojun Hong ${ }^{2}$, Qinyi Dai ${ }^{1}$, Tengyong Jiang ${ }^{3}$
}

\begin{abstract}
Desmin-related myopathy (DRM) is known to cause different types of cardiomyopathy. Late gadolinium enhancement cardiovascular magnetic resonance (CMR) has been shown to identify fibrosis in ischemic and nonischemic cardiomyopathies. We present a rare case of desmin-related hypertrophic cardiomyopathy, CMR revealed fibrosis in the lateral wall of the left ventricle. CMR is superior to conventional echocardiography for the detection of myocardial fibrosis in desmin-related cardiomyopathy, which may be useful to detect early cardiac involvement and predict the patient prognosis.
\end{abstract}

\section{Background}

Desmin is a primary element of the intermediate filament network in skeletal, cardiac and smooth muscle cells. Desmin-related myopathy (DRM) is an autosomally inherited skeletal and cardiac muscular myopathy mainly caused by mutations in the desmin gene [1]. DRM is characterized by progressive skeletal muscle weakness, cardiomyopathy, and cardiac conduction disease. The cardiac phenotype of DRM includes cardiac arrhythmias and different types of cardiomyopathy. However, cardiac symptoms are the leading cause of death in most patients [2]. Desmin-related cardiomyopathy is usually evaluated by echocardiography through functional and morphological changes. In recent years however, cardiovascular magnetic resonance (CMR) in combination with late gadolinium enhancement (LGE) imaging has been used to differentiate normal myocardium from a variety of myocardial diseases associated with necrosis or fibrosis. We describe a case of desminrelated hypertrophic cardiomyopathy with myocardial fibrosis detected by CMR, which has not been previously reported.

\section{Case report}

A 16 year old female presented to our cardiac department with progressive exertional dyspnea, palpitation, and skeletal muscle weakness for about two years. Two

\footnotetext{
* Correspondence: zhaoqi5000@vip.sohu.com

'Department of Radiology, Beijing Anzhen Hospital, Capital Medical

University, No. 2, Anzhen road, Chaoyang district, Beijing, China

Full list of author information is available at the end of the article
}

years ago she was diagnosed with hypertrophic cardiomyopathy by echocardiography. Her laboratory data showed creatine kinase (CK) $996 \mathrm{HU} / \mathrm{L}$, brain natriuretic peptide(BNP) $2634 \mathrm{Hpg} / \mathrm{ml}$. The electrocardiogram showed atrial fibrillation. At the referring hospital, skeletal muscle biopsies and mutation screening of the desmin gene had been performed according to the neurological workup of pathology.

Tranthoracic echocardiography showed symmetrical myocardial hypertrophy of interventricular septum and the wall of left ventricle. The wall motion was normal. Left ventricular ejection fraction (LVEF) was 66\%, left ventricular (LV) diastolic (DD) and systolic (SD) diameter was 40 and $26 \mathrm{~mm}$, respectively. The left atrium (LA) was dilated with diameter of $56 \mathrm{~mm}$.

CMR was performed with a $1.5 \mathrm{~T}$ Magnetom Sonata (Siemens Medical Systems). Fast-gradient-echo steadystate free precession cine demonstrated symmetrical wall thicken of interventricular septum and the lateral wall of left ventricle (Figure 1a). The left atrium was enlarged. Global and regional wall motion was normal (LVEF 63\%), in particular no segmental wall motion abnormality in the affected wall was observed. For myocardial tissue characterization, LGE imaging was performed with Gadolinium (Gd)-DTPA $0.2 \mathrm{mmol} / \mathrm{kg}$ BW (Magnevist, Bayer Schering Pharma, Berlin, Germany). The inversion recovery (IR) prepared 2-dimensional turboflash imaging revealed marked intramyocardial enhancement in the lateral LV wall (Figure 1b) indicating myocardial fibrosis. 


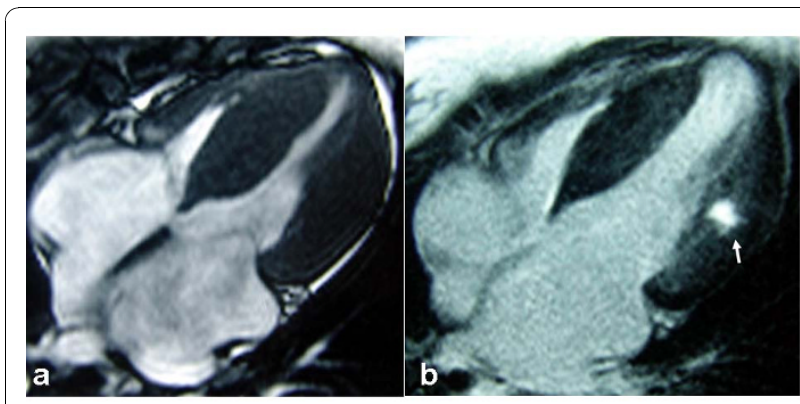

Figure 1 MRI imaging of the case. a A four-chamber view demonstrates symmetrical thickening of interventricular septum and the lateral wall of left ventricle. b A four-chamber view shows fibrosis involving the middle layer of left ventricle lateral wall (arrow).

The histopathological study of skeletal muscle was in line with the pathological pictures of desminopathy. Modified Gomori staining (MGT) showed multiple dark blue materials depositing in muscle fibers (Figure 2a), which were strong immunoreactivity to desmin antibody (Figure 2b). In addition, gene screening revealed a c.338_339delA_G deletion mutation in exon 1 of the desmin gene in this patient. This mutation caused a truncated protein at codon 115 (Q113fsX115) in the helix 1A domain (Figure 3).

\section{Discussion}

Desmin is the main intermediate filament protein expressed in skeletal, cardiac, and smooth muscle [3]. It interacts with other proteins to form a continuous cytoskeletal network that maintains a spatial relationship between the contractile apparatus and other structural elements of the cell, thus providing maintenance of cellular integrity, force transmission, and mechanochemical signaling. Primary desminopathies are caused by mutations in the desmin gene. This disease is characterized by an intracellular accumulation of insoluble protein aggregates eventually leading to cell death and replacement fibrosis [4].

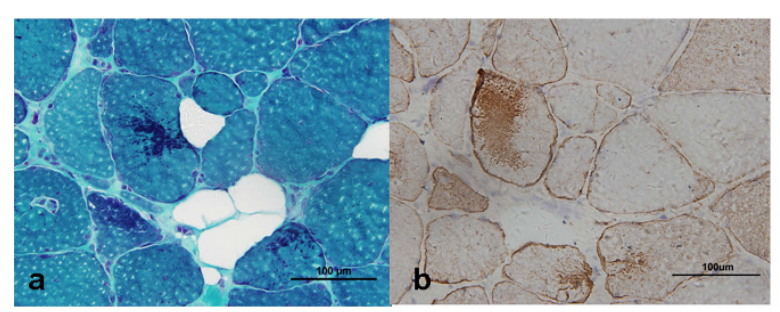

Figure 2 The skeletal muscle biopsy. Consecutive cryostat sections show some dark blue materials depositing in muscle fibers using MGT stain (a). The depositing materials are immunopositive to desmin antibody (b).

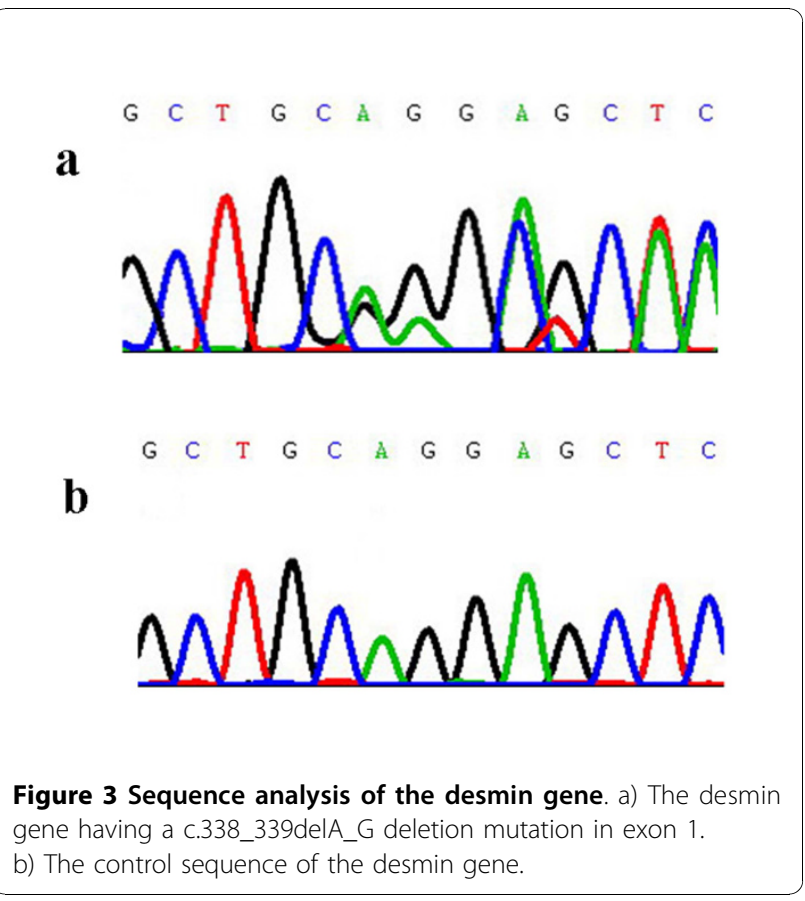

The clinical manifestationsis of desminopathies are quite broad including progressive skeletal myopathy, typically presents with lower and later with upper limb muscle weakness, sometimes slowly spreading to involve truncal, neck flexor, facial and respiratory muscles $[5,6]$. Different types of cardiomyopathy are also seen including dilated cardiomyopathy (DCM), restrictive cardiomyopathy (RCM), hypertrophic cardiomyopathy (HCM) and arrhythmogenic right ventricular cardiomyopathy (TRVC); Cardiac arrhythmias include various degrees of atrioventricular conduction block, and supraventricular and ventricular tachyarrhythmias.

Desmin-related cardiomyopathy is usually diagnosed with echocardiography through characteristically functional and morphological changes. Cardiomyopathy has been reported in up to $50 \%$ of desmin gene mutation carriers, with DCM and RCM being the most prevalent form, but HCM has seldom been reported. In recent years, CMR has evolved as the non-invasive reference standard for accurate and highly reproducible determination of cardiac function and morphology. But the study of desmin-related cardiomyopathy with CMR is really rare. Only one study systematically used CMR imaging to evaluate myocardial involvement in desminopathies. It revealed subtle focal hypertrophic myocardial changes in two desmin-related cardiomyopathy patients which were missed by echocardiography [7]. The reason may due to the high spatial resolution and clear delineation of endocardial borders of CMR, making it more sensitive for the detection of global and focal LV pathologies and subtle change of cardiac morphology than 
echocardiology that sometimes affected by limited acoustic windows.

Another important finding of CMR is the detection of fibrotic intramyocardial lesions by LGE imaging. It is the only direct and noninvasive imaging technique which can find myocardial fibrosis in cardiomyopathy. The finding of myocardial fibrosis is especially noteworthy since some desmin-related cardiomyopathies with myocardial fibrosis were not associated with global or focal systolic wall motion abnormalities and therefore might be missed by wall motion studies. The extent of myocardial fibrosis has been related to patient prognosis, including worsening of LV function and adverse outcome [8].

In conclusion, CMR was able to detect myocardial fibrosis in desmin-related hypertrophic cardiomyopathy. CMR may prove useful to detect early cardiac involvement and predict the patient prognosis of desminopathies.

\section{Consent}

Written informed consent was obtained from the patient for publication of this case report and any accompanying images. A copy of the written consent is available for review by the Editor-in-Chief of this journal.

\section{Author details}

${ }^{1}$ Department of Radiology, Beijing Anzhen Hospital, Capital Medical University, No. 2, Anzhen road, Chaoyang district, Beijing, China. 2Department of Neurology, Peking University First Hospital, Xishiku St 8\#, Xicheng District, Beijing,100034, China. ${ }^{3}$ Department of Cadiology, Beijing Anzhen Hospital, Capital Medical University, No. 2, Anzhen road, Chaoyang district, Beijing, China.

\section{Authors' contributions}

YH: Literature research, manuscript preparation and editing. ZZ: Revising the manuscript. DH: Carried out the molecular genetic and pathological studies. QD: Picture editing. TJ: Case collection.

All authors read and approved the final manuscript.

\section{Authors' information}

YH: MD, Deptartment of Radiology, Beijing Anzhen Hospital, Capital Medical University, Beijing, 100029, China.

\section{Competing interests}

The authors declare that they have no competing interests.

Received: 20 September 2010 Accepted: 18 November 2010 Published: 18 November 2010

\section{References}

1. Goldfarb LG, Park KY, Cervenáková L, Gorokhova S, Lee HS, Vasconcelos O, Nagle JW, Semino-Mora C, Sivakumar K, Dalakas MC: Missense mutations in desmin associated with familial cardiac and skeletal myopathy. Nat Genet 1998, 19:402-403.

2. Dalakas MC, Park KY, Semino-Mora C, Lee HS, Sivakumar K, Goldfarb LG: Desmin myopathy, a skeletal myopathy with cardiomyopathy caused by mutations in the desmin gene. N Engl J Med 2000, 342:770-780.

3. Lazarides E: Intermediate filaments as mechanical integrators of cellular space. Nature 1980, 283:249-255.

4. Fuchs $E$, Cleveland DW: A structural scaffolding of intermediate filaments in health and disease. Science 1998, 279:514-519.
5. Fischer D, Kley RA, Strach K, Meyer C, Sommer T, Eger K, Rolfs A, Meyer W, Pou A, Pradas J, Heyer C M, Grossmann A, Huebner A, Kress W, Reimann J, Schröder R, Eymard B, Fardeau M, Udd B, Goldfarb L, Vorgerd M, Olivé M: Distinct muscle imaging patterns in myofibrillar myopathies. Neurology 2008, 71:758-765.

6. Schröder R, Vrabie A, Goebel HH: Primary desminopathies. J Cell Mol Med 2007, 11:416-426.

7. Strach K, Sommer T, Grohé C, Meyer C, Fischer D, Walter MC, Vorgerd M, Reilich P, Bär H, Reimann J, Reuner U, Germing A, Goebel HH, Lochmüller H, Wintersperger B, Schröder R: Clinical, genetic, and cardiac magnetic resonance imaging findings in primary desminopathies. Neuromuscul Disord 2008, 18:475-482.

8. Kwon DH, Smedira NG, Rodriguez ER, Tan C, Setser R, Thamilarasan M, Lytle BW, Lever HM, Desai MY: Cardiac Magnetic Resonance Detection of Myocardial Scarring in Hypertrophic Cardiomyopathy Correlation With Histopathology and Prevalence of Ventricular Tachycardia. J Am Coll Cardiol 2009, 54:242-249.

doi:10.1186/1532-429X-12-68

Cite this article as: He et al:: Myocardial fibrosis in desmin-related hypertrophic cardiomyopathy. Journal of Cardiovascular Magnetic Resonance 2010 12:68.

\section{Submit your next manuscript to BioMed Central and take full advantage of:}

- Convenient online submission

- Thorough peer review

- No space constraints or color figure charges

- Immediate publication on acceptance

- Inclusion in PubMed, CAS, Scopus and Google Scholar

- Research which is freely available for redistribution 